\title{
Can a community-based nutritional screening and intervention programme improve clinical outcomes in Newcastle elderly care home residents?
}

\author{
C. G. Mountford ${ }^{1}$, N. P. Thompson ${ }^{1}$ and K. $\operatorname{Hart}^{2}$ \\ ${ }^{1}$ Newcastle Upon Tyne Hospitals NHS Foundation Trust, Freeman Road, Newcastle Upon Tyne NE7 7DN and \\ ${ }^{2}$ Department of Nutritional Sciences, University of Surrey Guildford GU2 7XH, UK
}

\begin{abstract}
Malnutrition affects more than 3 million people in the United Kingdom, of whom approximately $93 \%$ live in the community ${ }^{(1)}$. Elderly care home residents are at particular risk. This study aimed to establish the prevalence of malnutrition in Newcastle elderly care home residents and to evaluate the efficacy of a community-based nutritional screening and intervention programme in improving nutritional and clinical outcomes.

The study was approved as a service evaluation by the local NHS National Research Ethics Service and North Tyneside 1 Research and Ethics Committee. Five homes covering each of the wards within Newcastle upon Tyne Primary Care Trust were contacted and agreed to take part. Within homes the inclusion criteria for individual residents were that they were permanent residents and were able to eat and drink. Exclusion criteria included receipt of end of life or short-term respite care and existing use of oral nutritional supplements (ONS). Residents were allocated to one of three intervention groups according to their baseline Malnutrition Universal Screening Score (MUST) ( 0 , no active intervention; 1 , dietetic advice to increase oral intake; 2 or more, dietetic advice and prescribed ONS (220 ml, $1.5 \mathrm{kCal} / \mathrm{ml})$ twice daily). All subjects were followed up after 12 weeks. Measures of nutritional status, including anthropometrics, MUST score and mini nutritional assessment were recorded at baseline and 12 weeks. Perceived wellbeing was assessed using the Geriatric Depression Score (GDS) and hospital admission data for the period of the study intervention was compared to data for the previous year for each home.

Of 225 residents initially identified 205 were eligible for entry into the study ( $n=66$ males $n=139$ female) (mean age 84.2[8.5] years) with 173 undertaking the intervention phase and follow-up data available for 152. Over a third of the residents sampled were classified as malnourished or at risk of malnutrition $(37 \%)$ at baseline with mortality during the study period significantly associated with malnutrition risk (MUST 0 or $1,7.9 \%$; MUST $4,50 \%$; $p=0.004$ ). Only $54 \%$ of ONS prescribed were reported to be consumed. Nutritional status did not deteriorate in the majority $(86 \%)$ of residents during the study. However, there were also no clinically significant improvements in anthropometric measures following any study intervention or any significant effect of intervention group on anthropometric change. A significant association between change in MUST score and intervention group was identified $(p<0.001)$, with dietetic advice alone associated with the greatest improvement in 'MUST' score, and dietetic advice plus ONS the greatest deterioration. No significant changes in GDS or hospital admission rates were identified following any of the interventions.

This study did not find a positive effect of a 3-month intervention with oral nutritional supplementation and/or dietary advice on nutritional status, wellbeing or hospitals admission rates in elderly care home residents. The poorer prognosis in those with the most intensive intervention is likely to reflect their greater morbidity rather than a negative effect of the intervention per se, although issues of adequacy of the ONS prescription (in terms of both duration and quantity of intervention) and compliance with this should also be considered $^{(2)}$.

Screening for malnutrition is important to identify residents at risk of malnutrition however this study has identified potential cost savings via waste reduction rather than reduced morbidity or mortality. Dietetic input may slow the progression or improve nutritional status in some cases however results from this study do not support the widespread use of oral nutritional supplements in care home residents.
\end{abstract}

1. Russell CA \& Elia M (2010) Proc Nutr Soc 69, 465-9.

2. Edington J, Barnes R, Bryan F et al. (2004) Clin Nutr 23, 195-204. 